\title{
NOTAS PARA UNA REFORMA HOMOGENEA EN LA GESTION FINANCIERA: LA EXPERIENCIA DEL REINO UNIDO
}

\author{
Por EDUARDO ZOPICO GOÑI
}

Sumario: I. INTRODUCCIÓN: I. Reformas parciales interdependientes. II. GESTIÓN FINANCIERA EN LOS DEPARTAMENTOS BRITANICOS: I. Introducción. 2. Rasgos comunes de los planes ministeriales. III. Conclusiones.-Bibliografía.

I. Introducción: Reformas parciales interdependientes

Los Presupuestos Generales del Estado español para 1984 suponen el comienzo de una verdadera reforma presupuestaria. La intención inmediata de las autoridades financieras es la transformación del presupuesto clásico en un presupuesto por objetivos de tal manera que sirva como base para la toma racional de decisiones tanto a nivel de política presupuestaria como a nivel de gestión eficaz y eficiente de los recursos [1].

Aunque muy útiles y necesarios, los cambios propuestos en la Ley anual financiera no son por sí solos suficien- tes para poder cumplir los principios de eficacia y racionalidad económica contemplados en la Constitución Española, en la Ley de Procedimiento Administrativo y en la Ley General Presupuestaria. Cada día parece mayor la aceptación y necesidad de establecer en la Administración española unos modernos sistemas de dirección y gestión de los recursos y llevar a cabo una política de formación de personal que permita a los responsables de cada Ministerio u Organismo autónomo asignar los recursos bajo los principios mencionados.

Con este objetivo en mente se están haciendo también apreciables esfuerzos para implantar nuevos sistemas 
de contabilidad y control financiero que permitan of recer un seguimiento y unos estados financieros más fiables y apropiados a las necesidades del proceso decisorio.

No se pretende entrar aquí en un análisis del sistema financiero, ni criticar las reformas emprendidas en diversas áreas: presupuestaria, contable y de control, prácticamente en un periodo de gestación previo al de su implantación.

No obstante, es importante advertir desde estas líneas la necesidad de que estas reformas tengan muy en cuenta dos factores fundamentales: la interdependencia de las reformas mencionadas, entre si y de éstas con la formación del personal.

El desarrollo de cada una de las mencionadas reformas debe de ser homogéneo, coordinado y simultáneo al de los demás. Las tres áreas forman parte de un sistema único de información integral para el gestor. Este deberia disponer de una información, cuya fiabilidad fuera garantizada por un sistema moderno y flexible de control y no sólo de una información financiera, sino también de una información sobre los factores físicos realmente utilizados $y$, cuando la medición sea posible, sobre los resultados previstos y obtenidos con aquéllos. Sólo de esta forma estaría el gestor en una posición admisible para exigírsele una asignación presupuestaria óptima. Un fallo, o un adelanto o desfase, en cualquiera de las reformas provocaría un fracaso global de éstas $y$, por lo tanto, no se saldría de un circulo financiero vicioso que tiene como resultado unas decisiones sistemáticamente erróneas de las que no se puede culpar a ninguna fase en particular sin disculparla por el fallo de las que posteriormente le han facilitado su actuación. Es fundamental, por lo tanto, que los responsables de cada área mantengan constantemente una perspectiva global y común que debiera de culminar en la cobertura de unas necesidades de información básica para toda gestión que aspire a ser efica $z$ y eficiente.

Con respecto a la necesidad de formación del personal parece suficiente decir que es éste quien en última instancia ejecuta o implanta la reforma del sistema. Si no se tiene muy presente el vacio existente entre la capacidad actual del personal y la exigida por la reforma, es muy posible que los resultados de la misma, por muy congruente que ésta sea, queden anulados. Aunque se trate de una opinión no contrastada, no seria aventurado afirmar que la causa principal del fracaso de las reformas financieras iniciadas en los últimos años en las Administraciones públicas reside en una política inadecuada de formación de personal.

La preparación del personal es un requisito fundamental para poder llevar a cabo una modificación en los procedimientos clásicos de gestión y de control. Si se desea delegar competencias e implantar un control a posteriori que permitan una función directiva independiente, flexible y responsable, es necesario que, además de implantarse homogénea y progresivamente un sistema de gestión económico-financiera razonable exista un grado aceptable de confianza en la capacidad y competencia de los gestores. Esta estrategia es la seguida al menos por dos de los países sajones más avanzados en la carrera por la eficiencia y la eficacia en el sector público: Succia [2] y el Reino Unido. 
Con el fin de respaldar la importancia de las ideas expuestas y complementarla con nuevas aportaciones sobre estos temas es interesante volver la mirada hacia la experiencia vivida por la Administración pública del Reino Unido en el comienzo de esta década. En el siguiente apartado se resume el informe presentado en septiembre de 1983 por el primer ministro y el ministro de Hacienda ante el Párlamento británico ofreciéndose las lineas generales de la estrategia seguida por las autoridades británicas en su lucha contra la ineficacia e ineficiencia de su aparato administrativo [3].

\section{La gestión financiera en los de- partamentos británicos}

\section{1 INTRODUICCIÓN}

El Gobierno central británico viene utilizando una porción muy sustantiva de los recursos nacionales, y existe una presión constante de aumento del gasto como consecuencia del continuo ascenso de la demanda de servicios públicos. Se espera que el gasto público en 1983-84 represente un +3.5 por 100 del PIB. Para frenar este aumento y contener al mismo tiempo los impuestos y el endeudamiento público, es esencial que los recursos públicos sean utilizados eficientemente. Los departamentos necesitan ganarse la confianza pública y política persiguiendo programas relevantes y factibles, programas que funcionen, que proporcionen el máximo beneficio público al menor coste posible y que sean consistentes con los objetivos económicos y monetarios del gobierno. Deberían difundirse en todos los servicios del gobierno un sentido de la responsabilidad de obtener utilidad de los fondos administrativos.

Para combatir este problema el Gobierno británico inició, en mayo de 1982, un programa, el «Financial Management Iniciative", con el fin de mejorar la asignación, gestión y control de los recursos en el Gobierno central. Este programa se anunció en el Libro Blanco sobre «Eficacia y Eficiencia en el Servicio Públicon. EJ Libro Blanco trata de una amplia gama de medidas para mejorar la gestión administrativa en el Gobierno. El desarrollo del programa está en proceso y se irá informando de su resultado al Parlamento.

En este estudio se analiza el trabajo ya realizado por 31 departamentos (Ministerios y otros entes del sector público), y se ofrecen las líneas de actuación para los próximos años. La gestión financiera, según la utilización que aquí sé hace del término, es el enlace fundamental entre eficacia y eficiencia que debe caracterizar el trabajo diario de los servicios públicos y los objetivos económicos de la política gubernamental.

En mayo de 1982 se definieron los objetivos del programa como: «el promocionar en cada departamento una organización y un sistema en el que los directivos, a todos los niveles, tuvieran:

a) Una clara idea de sus objetivos, y los medios de valorar, y a ser posible, medir los resultados o actividades en relación a estos objetivos.

b) El establecimiento de responsabilidades para el mejor uso de los recursos.

c) La información (particularmente sobre costos), la formación y el 
acceso al asesoramiento de expertos para ejercer su funciones eficazmente.»

Estas líneas básicas de trabajo serán seguidas tanto por el Gabinete de ministros como por todos los jefes de los departamentos y directores de unidades administrativas, independientemente de su dimensión, ya sea grande o pequeña. No es la primera vez que se trabaja en el tema de mejorar rendimientos en los Ministerios. Se cuenta ya con experiencias anteriores, durante los años 60 y 70 , con resultados positivos. Estos son consecuencia de las técnicas modernas de gestión y los sistemas de información que el Gobierno introdujo siguiendo las instrucciones del informe Fulton de 1968. Sin embargo, se espera mejorar estos resultados ya que el nuevo plan tiene una perspectiva más amplia. En este caso no sólo se cubren los gastos de gestión administrativa sino también los gastos por programas. En este estudio se entiende por gastos administrativos los ocasionados por los recursos consumidos por el propio Gobierno $y$ por gastos de programas, los ocasionados por los consumidos en el exterior. Ejemplos de este último serían consecuencia de los contratos de compra, los beneficios de la Seguridad Social, la construcción de carreteras, etc.

Asimismo, el plan insiste en potenciar el desarrollo de los sistemas de información, ya que ésta es la base para la toma de decisiones en el Parlamento, en el Tesoro y en los Ministerios. Siempre que el centro o departamento reciba con regularidad información sobre el uso de los recursos y la eficacia de las actividades el gestor podrá ser sometido a un control de responsabilidad por su actividad y podrá tener una mayor participación en la elaboración de su presupuesto y una mayor libertad para ejecutarlo. Esta delegación de autoridad le permitirá mejorar la eficacia y eficiencia de su servicio y ser más responsable por su actividad.

Una mejor información tendría poco valor sin unas instalaciones adecuadas para su tratamiento y unos técnicos especializados para su uso. Uno de los caracteres esenciales del plan es el desarrollo y mejora del aparato administrativo, que permita una revisión sistemática de los gastos, los objetivos, los recursos dedicados a ellos y el grado de éxito obtenido. La otra gran caracteristica sería la formación de gestores a todos los niveles.

Los costes de los trabajos hasta ahora realizados dentro de este plan han sido relativamente pequeños $y$ en su mayor parte ocasionados por motivos de investigación y planificación. En el futuro los costes directos del plan aumentarán considerablemente (35 millones de libras en los próximos dos años). Este gasto se invertirá en la contratación de personal y la adquisición de computadoras, terminales y "software".

La ejecución del plan propuesto en el "Financial Management Iniciative" necesita tiempo. Los nuevos sistemas tienen que ser elaborados en detalle, sometidos a un rodaje y, por último, modificados. El personal tiene que familiarizarse con los nuevos procesos y formarse en relación con las nuevas técnicas. A través de la Unidad de Gestión Financiera (FMU), la Oficina de Gestión y Personal (MPO) y el Tesoro continuarán en estrecho contacto entre sí y con los departamentos gestores a medida que el trabajo se 
vaya realizando. Algunos Ministerios han sugerido al Tesoro la participación en Comités y grupos de trabajo conjuntos para llevar a cabo el seguimiento de la ejecución del plan y permitir la difusión de ideas, tanto teóricas como prácticas, en los distintos Ministerios. Con el fin de proyectar el plan más allá de la duración de una legislatura, se contará con la opinión de los sindicatos de funcionarios que serán consultados por los propios $\mathrm{Mi}$ nisterios, por el Tesoro y por el MPO.

En el Libro Blanco se sintetiza el plan de trabajo bajo cuatro apartados:

a) Sistema de información para los ministros y altos directivos.

b) Gestión de los gastos administrativos.

c) Gestión de los gastos de programas.

d) Políticas de gestión y formación del personal.

Los resultados de la aplicación del plan serán publicados por el Gobierno en julio de 1984.

\section{2 Rasgos Comunes de los Planes MINISTERIALES}

Sistemas de información para los ministros y' altos directivos

Los planes se centran en el sistema de información y equipos necesarios para facilitar a los ministros y altos directivos la formulación de objetivos, el establecimiento de prioridades y la asignación de recursos disponibles de cada departamento, asegurar un conocimiento de prioridades y permitir la adaptación al cambio en todos los niveles.

El sistema de información de gestión para los ministros utilizado en el
Departamento de Medio Ambiente (MINIS), ha sido uno de los pioneros en este campo. Inicialmente fue desarrollado para permitir al secretario de Estado responder a la pregunta: "QQuién hace qué, por qué y a qué coste?». El sistema ha continuado desarrollándose en estos últimos cuatro años. Los tres rasgos que lo definen son:

1. La preparación sumaria de los planes y las actividades del departamento y sus costes.

2. La evaluación de estos planes $y$ actividades en reuniones del ministro con los altos directivos.

3. El continuo seguimiento de las medidas acordadas.

Otros Ministerios están desarrollando sistemas de información similares al MINIS. Cada uno de ellos es analizado posteriormente en la segunda parte del estudio. En general, en todos los Ministerios, los sistemas de información abarcan los gastos administrativos $y$, en la mayor parte de ellos, los gastos por programa. En la mayoria de los casos la información es elaborada en las oficinas de la Subsecretaría de cada departamento. Los sistemas de información introducidos en muchos de los departamentos proporcionan una información directa a los gestores que ocupan puestos de carácter ejecutivo, sobre los costes, objetivos y medidas de actividad.

Cada Ministerio necesita reorganizarse para llevar a cabo el control y revisión de sus programas y costes administrativos. Según la naturaleza de las actividades del departamento, ambas informaciones se podrán cubrir mediante un sistema único o varios estrechamente relacionados. 
Muchos Ministerios están introduciendo nuevas instalaciones o mejorando las que tenían. Como modelo general se ha establecido un Comité, presidido por el secretario permanente, que informa al ministro y supervisa la ejecución de sus decisiones.

\section{Gestión de los gastos administrativos}

Los planes proponen la división del trabajo de los departamentos en bloques apropiados y en centros de costes dentro de cada uno de estos bloques. Esto permitirá responsabilizar a los directores de la gestión de los 'recursos bajo su control y de los resultados alcanzados mediante procedimientos, sistemas de información y medidas para el establecimiento de objetivos contra los cuales la actividad deberá ser juzgada.

En la parte II del estudio se describe el futuro desarrollo de estas innovaciones en los distintos Ministerios. Se están preparando nuevos esquemas para el desarrollo de otras experiencias. Se está indentificando a los responsables de las partidas presupuestarias. Se están desarrollando nuevos sistemas de información y contabilidad, de tal manera que los costes y resultados de cada unidad de gestión sean conocidos con regularidad, y por último, se está familiarizando a Tos gestores con los nuevos procedimientos y sistemas mediante una apropiada formación.

El desarrollo de los sistemas de información irá generalmente acompañado de un cierto desarrollo en el proceso presupuestario, de tal manera que los gestores puedan tomar decisiones sobre el uso de los recursos administrativos dentro de la política y procedimientos que aplican a su trabajo.

Todas estas reorganizaciones supondrán una mayor participación del gestor en el proceso presupuestario administrativo y delimitarán la responsabilidad de controlar su costes y resultados una vez que el presupuesto haya sido fijado. El gestor necesita un claro conocimiento sobre los fondos y demás recursos disponibles para su servicio, la información oportuna de los costes de tal manera que se sepa lo que está siendo gastado en cada ejercicio, y las medidas de actividad adecuadas a las que poder relacionar los gastos.

La parte segunda del informe muestra una variedad de estrategias departamentales. Desde la formación de una cadena completa de centros de costes y responsabilidad (Ministerios de Medio Ambiente y Transportes) hasta la reorganización mediante una delegación considerable y específica de autoridad discrecional en los directores de grandes unidades autónomas.

El número de responsables de partidas presupuestarias en un determinado departamento y la amplitud de la delegación de responsabilidad para la administración de los recursos, reflejarán su naturaleza y su cometido. Así, por ejemplo, el Ministerio del Medio Ambiente, donde la oficina del subsecretario está compuesta por unas 150 personas, el responsable presupuestario típico es un secretario adjunto que tiene a su cargo entre 10 ó 40 personas. Por el contrario, el Ministerio de Salud y Seguridad Social con aproximadamente 60.000 funcionarios y más de 500 oficinas, ha escogido la oficina local como unidad básica del proceso presupuestario. 


\section{Gestión de los gastos de programas}

Los gastos por programas son más variados que los gastos administrativos. Algunos son tarea directamente de los Ministerios, mientras que otros son responsabilidad de las autoridades locales, corporaciones públicas y otros entes públicos. El plan global de gastos del Gobierno es revisado y orientado cada año dentro del examen de gastos públicos (PES).

Las líneas comunes del desarrollo de la gestión de los programas son:

a) Clarificar la extensión y la naturaleza de la responsabilidad de la dirección en cada departamento. Esto se deberá reflejar en los planes de cada Ministerio teniendo en cuenta el sistema de información elegido, los costes corrientes y los del propio programa, las alternativas, etc. Esta información será utilizada a lo largo de la vida de todo el programa, pudiendo ser la duración de algunos hasta de diez años.

b) Mejorar la evaluación de los proyectos y en concreto el análisis del coste-eficacia. En algún Ministerio, como el de Comercio e Industria, se ha puesto un mayor énfasis en este último, especialmente para estrategias de asistencia industrial, y en la introducción de objetivos cuantificados con el propósito de valorar a posteriori el grado de consecución de éstos.

c) Clarificar responsabilidades y fomentar relaciones fuera del limite estricto de cada departamento. La primera responsabilidad para la ejecución de los programas radica en las entidades afectadas que debieran adecuar los planes de reorganización para asegurar que sus objetivos son consecuentes con los marcados por el Mi- nisterio. Los directores de programa de cada departamento deberán aprobar estas reorganizaciones, asegurar que las entidades disponen de sistemas de dirección efectivos, y examinar sus actividades en relación con los objetivos. Entre otros, los planes del Ministerio de Medio Ambiente, del Ministerio de Educación y. Ciencia y del Ministerio de Salud y Seguridad Social incluyen acciones de este tipo.

Un informe reciente del Ministerio de Salud y Seguridad Social sobre el mantenimiento de la salud y sus costes, describe las medidas que han sido tomadas para fortalecer la dirección financiera y mejorar la eficiencia de los servicios; entre éstas se incluye la delineación de la estructura de directivos y de un nuevo sistema de revisión de responsabilidades basado en el desarrollo de indicadores de actividades y mejora en general de los sistemas de información.

Las autoridades locales son las responsables directas ante sus electores y contribuyentes de asegurar que sus servicios sean adecuados económica y eficientemente. Sin embargo, el Gobierno central está también implicado en muchos aspectos del trabajo de dichas autoridades locales y su actividad les afecta en muchos sentidos. El Gobierno central, por lo tanto, necesita fomentar y ayudar a las autoridades locales para mejorar constantemente su eficacia y eficiencia. El Gobierno también necesita asegurar que sus propios medios de influencia sobre las autoridades locales a través de controles, subvenciones, préstamos, etc., son manejados de tal manera que individual y colectivamente promuevan la buena práctica del Gobierno local. 
Durante los cuatro últimos años, el Gobierno central ha animado a otras autoridades a realizar un seguimiento más estrecho de sus propias actividades, por ejemplo, mediante la comparación y publicación de los costes de ciertos servicios. Además, se han constituido unas comisiones de Auditoría para promocionar la buena práctica en los Gobiernos locales y para promocionar y desarrollar estudios comparativos y auditorías financieras. Se ha reformado también el sistema de subvenciones y de control de gastos de capital, dando una atención especial a la mejora del flujo de información sobre asuntos financieros de los Gobiernos locales de tal manera que se mejore la política macroeconómica del país.

El Ministerio de Medio Ambiente y la Oficina de Escocia están actualmente discutiendo junto con otros $\mathrm{Mi}$ nisterios y Gobiernos locales cómo debería de mejorarse el funcionamiento del Gobierno central y local. Se está prestando atención al reforzamiento de la planificación financiera a medio plazo de gastos de capital, a la eficiencia de las medidas tomadas para ejecutar las subvenciones, $y$ al desarrollo de medidas y resultados.

El Gobierno central tiene ciertos poderes y deberes especificos en relación con determinados servicios. Por ejemplo, en relación con la seguridad ciudadana el Ministerio del Interior tiene legalmente establecido el deber de promocionar la eficiencia de los servicios de policía. Esto no sería apropiado en otros servicios locales tales como la educación, donde la ayuda del Gobierno central es proporcionada a través de subvenciones no especificas. Sin embargo, este tipo de subvenciones permite también una cierta influencia del Gobierno central. Así, por ejemplo, los planes del Ministerio de Educación incluyen el desarrollo de:

a) La relación entre los gastos de educación de Gobiernos locales y la valoración hecha por el Gobierno central de las necesidades de subvenciones para los propósitos de aquéllos.

b) Los medios de valoración de resultados y recursos.

\section{Repercusiones en la Dirección de personal}

Los planes anteriormente mencionados no serán efectivos sin unos cambios y mejoras complementarias en la dirección y formación del personal.

Para muchos directivos el personal es el factor más importante y el de mayor coste; por el cual son responsables. El MPO ha publicado un informe sobre la revisión del trabajo del personal en nueve Ministerios paralelamente con la elaboración de los planes de dirección financiera. Este informe apoya también la idea de responsabilizar a los directivos de la gestión diaria del personal.

Formación del personal para la gestión financiera

Los grupos destinatarios más importantes de esta formación sobre gestión financiera de los recursos son:

a) El personal que posiblemente en un futuro ocupe puestos de dirección para los que se requiera un conocimiento del presupuesto y prácticas financieras, el sistema financiero en general del Gobierno y los sistemas 
operativos propios de cada departamento.

b) Los expertos en estos sistemas que trabajan en divisiones financieras $y$ otras actividades relacionadas.

c) El personal que tenga una responsabilidad significativa en la dirección de los recursos.

Los departamentos, la Escuela del Servicio Civil y otras entidades proporcionarán directamente una formación adecuada. La Escuela del Servicio Civil se concentra en los conceptos de aplicación general en el Gobierno, la formación de los oficiales superiores, y la organización y formación en el área de contabilidad y auditoría. La demanda de asistencia a cursos en valoración de inversiones, finanzas, contabilidad y auditoría interna experimenta un gran aumento y el número de dias-estudiante proporcionado se duplicará en los próximos dos años.

La Escuela introdujo un curso superior de finanzas en $1982 / 83$ para los oficiales superiores que es obligatorio para aquellos que vayan a ser nombrados directores financieros (Principal Financier Officer) en cada departamento. Este curso está siendo objeto de un mayor desarrollo y por el momento incluye los siguientes módulos: no.

- Gestión financiera del Gobier-

- Contabilidad pública.

- Contabilidad privada.

- Contabilidad para la gestión.

- Asignación de recursos.

- Evaluación de inversiones.

- Medición de actividades.

- Uso de computadoras en la gestión financiera. dad.
Además, la Escuela está preparando una serie de seminarios para reunir al personal que tiene responsabilidades muy relevantes respecto al gasto administrativo y por programas. Esto ayudará a que se compartan ideas o planes y en gran medida complementará el trabajo realizado por el Tesoro y el MPO.

Con respecto a la formación en auditoría interna, se ha establecido un programa bajo los estándares establecidos por el Tesoro. Con el fin de cubrir rápidamente la demanda, un número de Escuelas Politécnicas están organizando cursos en cooperación con la Escuela del Servicio Civil. En cuanto a la formación contable se han organizado cursos no sólo en estas Escuelas Politécnicas sino también en firmas privadas de contabilidad así como en los centros de formación del Departamento de Defensa.

La mayor parte de la formación será proporcionada por el propio personal de cada departamento, concentrándose ésta en la aplicación práctica de la gestión financiera de los propios sistemas. En el Ministerio de Medio Ambiente y de Transportes por ejemplo, se han organizado seminarios para todos los directores de centros de coste y oficiales relacionados que vayan a ser responsables de la operación diaria del sistema. En el Ministerio de Salud y Seguridad Social la introducción de centros de coste fue precedida por un proceso de formación y ésta continuará en la medida que sea necesario para el proceso presupuestario.

Por otro lado, no ha dejado de considerarse la necesidad de desarrollar programas de formación en técnicas y especialidades de cada departa- 
DA-1984, núm. 200. EDUARDO ZAPICO GOÑI. Notas para una reforma homogénea en la gestión fin...

mento. Por ello la Oficina del Interior ha organizado un curso sobre las técnicas de valoración de inversiones en cooperación con la ESC.

\section{Problemas prácticos comunes}

Hay algunos problemas que se presentan comunes a todos o a la mayor parte de los Ministerios. Quizá los más importantes son aquellos que se refieren a la delegación de responsabilidades. Por ejemplo:

a) ¿Cómo integrar el control del número de funcionarios en el presupuesto de gastos? Ya que los costes de personal son con mucho los de más peso dentro de los costes administrativos, sería deseable incluirlos en cada presupuesto, de tal manera que se mantenga un control serio sobre el tamaño del servicio.

b) ¿Cómo equilibrar la flexibilidad interna de cada presupuesto (con el fin de aumentar la responsabilidad del director correspondiente en el uso de sus recursos) con la flexibilidad entre las distintas unidades presupuestarias (permitiendo en cierta medida la maniobrabilidad dentro del departamento a lo largo de todo el año)?

Otros problemas comunes son:

c) Determinar cuál sería la duración óptima del ciclo presupuestario.

d) Desarrollar los indicadores adecuados para la medición de los resultados.

e) En caso de que exista una red de oficinas locales, ¿cómo podría asegurarse que su personal consiga alcanzar los objetivos prioritarios si sus tareas son especificadas por un número diferente de divisiones en la oficina central?
El Tesoro y el MPO se mantendrán en contacto en relación al progreso experimentado respecto a estos problemas y asegurarán que las experiencias útiles sean difundidas. En este sentido $y$ en otros de distinta naturaleza, ambos serán ayudados por las unidades de gestión financiera.

La inspección de personal, la auditoría interna y la gestión de los servicios

La confianza en la competencia de los gestores y en los sistemas es una condición fundamental para posibilitar la delegación de autoridad. El cambio en los sistemas de control no debe suponer una pérdida de dicho control. Los planes de algunos departamentos incluyen la implantación de unos servicios de inspección y asesoramiento. La inspección de personal examinará el número y.el nivel del personal en relación con el trabajo que deba de hacerse. La auditoría interna velará por la eficacia del control interno del departamento complementando la responsabilidad primaria de los gestores. Ambos proporcionarán una mayor garantía al trabajo realizado por la oficina contable de cada departamento. Ambos también proporcionarán asesoramiento a los gestores complementando sus servicios.

Un tema común a los planes de reformas en los Ministerios es la mayor coordinación de las unidades afectadas con el fin de maximizar su eficacia. El Ministerio de Defensa ha considerado oportuno reunir estas tres funciones en la nueva organización. Otras entidades han creado unos comités de coordinación presididos por el director financiero de cada departamento (Principal Financier Offi- 
DA-1984, núm. 200. EDUARDO ZAPICO GOÑI. Notas para una reforma homogénea en la gestión fin...

Crónicas

cer) para armonizar los problemas y elaborar trabajos interdisciplinarios cuando sea necesario.

Muchas de las unidades de auditoría interna ya han sido reestructuradas, la preparación del personal va mejorando a través de los programas de formación, y el Tesoro ha publicado un manual de auditoría interna enunciando los estándares y líneas generales de una buena gestión e inspección del personal.

El programa de eficacia para 1983 incluye una revisión, centralmente coordinada, en seis departamentos sobre sus capacidades de inspección, revisión y consulta. Este trabajo quedará terminado a principios de 1984 . Igual que en el estudio de la revisión de los costes de funcionamiento, se espera que las conclusiones de este trabajo sean útiles para muchos más departamentos que los que han tomado parte en su elaboración.

Implicaciones en el trabajo del Tesoro y del $M P O$

El Tesoro debe asesorar al Consejo de Ministros sobre la cuantía total del gasto público y su distribución de acuerdo con la política económica del gobierno. También debe asegurar que el gasto público del ejercicio esté ajustado a lo previsto. Por estas razones se regula la realización de un análisis de costes y resultados de cada uno de los programas de gasto y una información oportuna y fiable del flujo del gasto. El resultado de la evaluación de los gastos deberia estar a disposición del Tesoro y el trabajo se ha establecido de tal manera que los sistemas de información financiera puedan ayudar a mejorar la oportunidad y fiabilidad de los datos y previsiones que actualmente son proporcionados al Tesoro.

El diálogo mantenido entre el Tesoro y los Ministerios gestores sobre la revisión del gasto público y los importantes aumentos en nuevas propuestas de gasto deberian ser más fructíferos en función de los datos recibidos por los nuevos sistemas de información ministeriales. Simultáneamente, a medida que estos sistemas vayan mejorando, tanto el Tesoro como el MPO debería de aumentar su confianza en la efectividad operativa de los departamentos y reducir la necesidad de consultas previas sobre asuntos menores. La normativa existente sobre delegación de autoridad por parte del Tesoro y el MPO a los departamentos gestores está siendo revisada teniendo presente lo anteriormente dicho y el proceso continuará en la medida que los Ministerios desarrollen sus sistemas.

Por lo tanto, el Tesoro y el MPO se preocuparán en adelante y de una forma creciente de la eficacia de los sistemas ministeriales y de la garantía de los flujos de información más que del trabajo en detalle sobre problemas menores. En las áreas principales, tales como la evaluación de inversiones, se están desarrollando unas líneas generales y estándares de inspección de personal y auditoría interna para facilitar a los departamentos la gestión de los recursos. El Tesoro y el MPO vigilarán de forma continuada la aplicación de estas medidas.

Cobertura de las necesidades del Parlamento y' del ciudadano

La introducción en un Ministerio de un sistema de control presupuesta- 
rio descentralizado podría alterar el detalle de las éstimaciones presentadas anualmente ante el Parlamento. Cuando el trabajo de los departamentos haya establecido sus necesidades, el Tesoro elevará las propuestas de asignaciones a los comités parlamentarios.

El desarrollo de la estructura, contenido y forma del informe anual de gastos públicos y las "Estimaciones de Ofertan, tendrán en cuenta la opción del Comité de Procedimientos y las necesidades de los comités especialmente relacionados con cada departamento así como las del Tesoro, el Departamento de Auditoría y el Comité de Contabilidad Pública. Estos documentos serían el punto de partida para examinar la actividad del departamento con más profundidad. En concreto, se pretende que el Libro Blanco contenga unos estados más claros de la política de objetivos a la que se dirige el gasto y de los indicadores de actividad y resultados cuando esto sea posible. La intención es que haya una relación más estrecha entre los gastos públicos del Libro Blanco, las estimaciones y los sistemas de información que los departamentos están desarrollando para la gestión de los programas, actividades y costes.

\section{Conclusiones}

Como consecuencia más importante de la naturaleza interdependiente de las reformas de las distintas áreas de la gestión financiera, incluida la formación de personal, parece muy aconsejable seguir una estrategia de implantación progresiva de cada una de ellas. Sería muy arriesgado que se diera bruscamente una mayor libertad a la función directiva de un organismo sin haberse homologado previamente sus sistemas de información y gestión, así como la capacidad de su personal.

Habiendo ya comentado de forma global e interrelacionada esta característica de interdependencia y progresividad de las reformas proyectadas parece oportuno que para terminar este trabajo destaquemos otras sugestivas aportaciones de la experiencia britảnica.

Uno de los puntos diferenciados es el prolongado período de tiempo durante el que la Administración del Reino Unido ha estado sometida a análisis para la implantación de sistemas modernos de gestión. El nuevo programa de reforma aunque se basa en fructíferas reformas de la década de los sesenta y setenta lleva cerca de dos años principalmente en fase de investigación y planificación.

En segundo lugar, y no menos importante, es muy destacable la adaptabilidad con que se impone la reforma de la gestión financiera a los distintos Ministerios. Aunque similares en naturaleza, cada Ministerio ha adoptado una estructura y unos sistemas de información y gestión adecuados a sus necesidades y posibilidades. Así, por ejemplo, el responsable presupuestario puede ser desde un secretario adjunto hasta un jefe de una oficina local. En este caso todo depende del de la organización, tamaño y otros factores variables.

Por otro lado, y aunque en cierta medida ya se ha mencionado, es interesante destacar la estrategia seguida para llevar a cabo la formación del personal en materia financiera. En efecto, las autoridades británicas es- 
tán desarrollando un plan de cursos y seminarios dirigidos a la formación del personal que ocupe o pueda en el futuro ocupar puestos directivos o de cierta responsabilidad, relacionados con el presupuesto $\mathrm{y} / \mathrm{o}$ gestión financiera, en el que colaboran una larga serie de entidades: La Escuela del Servicio Civil, el Tesoro, las oficinas de personal de cada uno de los departamentos gestores e incluso las escuelas politécnicas. Las materias impartidas cubren prácticamente las áreas relevantes de la gestión financiera: Contabilidad, Evaluación de Proyectos y Asignación de Recursos, Informática, Medición de Actividades, etc.

Es importante también destacar la acción conjunta y creciente del Tesoro y la Oficina de Gestión y de Perso- nal para realizar el seguimiento de la eficacia y eficiencia de los sistemas de gestión financiera de cada Ministerio. Esta acción está relacionada con la reducción de la necesidad de consultas previas sobre asuntos menores y delegación de competencias desde estos dos centros directivos a los centros gestores.

Por último, y aunque pueda parecer elemental y de puro sentido común, el hecho de que los responsables del programa de reforma de la gestión financiera hayan estudiado y planeado la adquisición y puesta en marcha del equipo e instalaciones necesarias para almacenar y transportar los flujos de información esperados tras la reforma nos hace pensar que ésta va totalmente en serio.

\section{BIBLIOGRAFIA}

[1] Secretaria General de Presupuesto y Gasto Público: Informe económico y financiero. Presupuesto 1984. Ministerio de Economía y Hacienda, Madrid, 1984, pp. 149 y ss.

[2] The National Audit Bureau: The State Economic Administrative Sistem. NAB, Stockholm, 1976.

[3] Prime Minister and Chancellor of the Exchequer: Financial Management in Government Departaments. Her Majesty's Stationery Office. London, 1983. 
DA-1984, núm. 200. EDUARDO ZAPICO GOÑI. Notas para una reforma homogénea en la gestión fin...

DA-1984, núm. 200. EDUARDO ZAPsICO GOÑI. Notas para una reforma homogénea en la gestión fin... 
DA-1984, núm. 200. EDUARDO ZAPICO GOÑI. Notas para una reforma homogénea en la gestión fin...

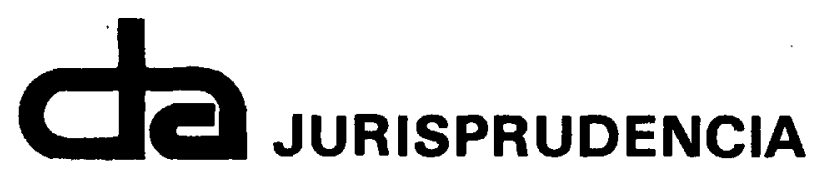

- Constitucional

- Contencioso-administrativa

DA-1984, núm. 200. EDUARDO ZAPICO GOÑ்l. Notas para una reforma homogénea en la gestión fin... 
DA-1984, núm. 200. EDUARDO ZAPICO GOÑI. Notas para una reforma homogénea en la gestión fin...

DA-1984, núm. 200. EDUARDO ZAPICO GOÑI. Notas para una reforma homogénea en la gestión fin... 